\title{
Failure Rate of Adenoidectomy and Reasons of Failure in the Short Term
}

\author{
Süay Özmen ${ }^{1}$, Ömer Afșin Özmen ${ }^{2}$ \\ ${ }^{1}$ Otorhinolaryngology Clinics, Inegöl Government Hospital, Bursa, Turkey \\ ${ }^{2}$ Department of Otorhinolaryngology, Medical Faculty, Uludağ University, Bursa, Turkey \\ Email: suayozmen@yahoo.com
}

Received March 12, 2012; revised April 20, 2012; accepted May 11, 2012

\begin{abstract}
Objectives: The aim of the present study was to analyze the recovery rate of symptoms in patients with adenoid hypertrophy and investigate the role of nasal pathologies, allergy and gastroesophageal reflux (GER) as the reason of failure in patients with persistent symptoms. Patients and Methods: Patients undergoing adenoidectomy were enrolled in this study. There were 58 males and 42 females, aged between $1-13$ years (mean \pm SD: $4.9 \pm 2.2$ years). The parents of each child were questioned about the following symptoms; apnea, nasal obstruction, mouth breathing, snoring and nasal discharge. Presence or absence of nasal obstruction due to septal deviation and/or chonchal hypertrophy was noted. All children were evaluated for GER by upper gastrointestinal endoscopy or $\mathrm{pH}$ monitorization and for allergy by specific IgE analysis or skin prick test. Three months after the operation the children were re-examined and their parents were interviewed about persistent symptoms. Patients with persistent symptoms were re-evaluated with rigid endoscopy for residual adenoid vegetation. Results: Apnea was cleared in the entire group postoperatively. However, 9 patients complained of nasal obstruction, 16 patients had mouth breathing, 9 patients had snoring and 17 patients continued to have nasal discharge two months after the operation. Statistical assessment showed a significant difference for each symptom between the pre- and postoperative level $(\mathrm{p}<0.001)$. In the re-examination of the patients with persistent symptoms, none of them were found to have adenoid vegetation greater than grade 1 . However, $71 \%$ of these patients had nasal pathology, 50\% had allergy and 50\% had GER. Conclusions: Adenoidectomy alone is an effective treatment for nasal obstruction and obstructive sleep symptoms in children. Persistent obstructive symptoms are usually due to nasal pathology and allergy.
\end{abstract}

Keywords: Adenoidectomy; Nasal Obstruction; Snoring

\section{Introduction}

Adenoidectomy is among the most common pediatric operations [1]. Adenoidectomy is recommended for the treatment of upper airway obstruction and recurrent or chronic adenoiditis, which may present as chronic rhinosinusitis or chronic otitis media with effusion. Awareness of the potentially serious sequelae of upper airway obstruction has increased, and more adenoidectomies are now being done for this indication. Complications include learning difficulties, behavioral changes, and, in severe cases, failure to thrive, pulmonary hypertension, and secondary right-sided cardiac hypertrophy. Adenoid obstruction may also lead to an open anterior bite and "adenoid facies" caused by the maxillary and mandibular structural changes that are believed to occur secondary to chronic mouth breathing [2].

Different studies reported $70 \%$ to $100 \%$ improvement in quality of life (QOL) and various symptoms following adenotonsillectomy [3-7]. There are some limited reports that adenoidectomy produces benefits in the short term [8-9]. However, evidence about the benefit after the first few months is lacking. There are a number of studies that report the incidence of allergy and GER in patients who have adenoid hypertrophy [10-12].

The aim of the present study was to analyze the recovery rate of symptoms in patients with adenoid hypertrophy and investigate the role of nasal pathologies, allergy and GER as the reason of failure in patients with persistent symptoms.

\section{Patients and Methods}

One hundred children undergoing adenoidectomy alone (i.e., without tonsillectomy and/or ventilation tube insertion) were enrolled in this study. There were 58 males and 42 females, aged between $1-13$ years (mean \pm SD: $4.9 \pm 2.2$ years). The design of the study has been approved by the Ethical Committee of Bursa Dörtçelik Children Hospital. After informed consent was obtained 
from the parents, patients admitted for adenoidectomy. Exclusion criteria were: underlying chronic illness or bleeding disorder or craniofacial defects, immunodeficiency, grade 3 - 4 tonsillar hypertrophy. The parents of each child were questioned about the following symptoms; apnea, nasal obstruction, mouth breathing, snoring and nasal discharge.

A routine ENT examination was performed on all children. Adenoidectomy was recommended for adenoid hypertrophy. Adenoid hypertrophy was evaluated by $0^{\circ}$ 2.7-mm rigid nasal endoscope (Karl Storz, Germany) which was performed 10 minutes after topical application of a $1: 1$ solution of $10 \%$ xylocaine spray and $1 \%$ ephedrine to the nostrils. The degree of adenoid obstruction was evaluated as follows [13]:

Grade 1: no or minimal adenoid tissue $(0 \%-25 \%$ obstruction);

Grade 2: small amount of adenoid tissue $(26 \%-50 \%$ obstruction);

Grade 3: large amount of adenoid tissue that might cause symptomatic nasal obstruction $(51 \%-75 \%$ obstruction);

Grade 4: obstructing adenoid tissue $(76 \%-100 \%$ obstruction).

Tonsillar hypertrophy was graded as follows $[14,15]$ :

Grade I: tonsils located in the tonsillar fossa, barely seen behind the anterior pillars;

Grade II: tonsils visible behind the anterior pillars;

Grade III: tonsils extend three-quarters of the way to the midline;

Grade IV: tonsils completely obstruct the airway.

Presence or absence of nasal obstruction due to septal deviation and/or chonchal hypertrophy was noted.

All children were evaluated for GER by upper gastrointestinal endoscopy or $\mathrm{pH}$ monitorization and for allergy by specific $\operatorname{IgE}$ analysis or skin prick test.

The children were treated using conventional adenoidectomy technique. All operations were performed under general anesthesia by the same otolaryngologist (SO). The patients were positioned supine in Rose position and the neck was extended with a shoulder-roll placed under the shoulders. The adenoidectomies were performed using adenoidectomy curettes. A Boyle-Davis gag was inserted. The palate was palpated to exclude a possible submucosal cleft. Complete removal was confirmed by digital palpation or mirror examination. After adenoidectomy, pressure was applied to nasopharynx by tonsillar pack for three minutes. The use of cautery was rarely necessary for hemostasis. The patients were discharged the same day after the operation. They routinely received 7 days of postoperative ampicillin or amoxicillin.

Three months after the operation the children were re-examined and their parents were interviewed about persistent symptoms. Patients with persistent symptoms were re-evaluated with rigid endoscopy for residual adenoid vegetation.

All statistical analyses were performed by using SPSS for windows v.16 (SPSS inc. Chicago, USA). McNemar test was used to check whether there was a significant difference between the preoperative and postoperative symptoms or not. In order to evaluate the impact of nasal pathology, allergy and GER on the postoperative symptom rates, Fisher's exact test was employed. A p value less than 0.05 was accepted as statistically significant.

\section{Results}

In the preoperative assessment, most of the children had nasal obstruction, mouth breathing and snoring (Table 1). Apnea was present in 43 patients. Half of the patients complained of nasal discharge. In the physical examination, 30 children had grade 2, 7 children had grade 3, 63 children had grade 4 adenoid hypertrophy. Seventy-eight patients had grade 1 tonsil hypertrophy, 22 patients grade 2 tonsil hypertrophy. Nasal anatomy was distorted in 28 patients. Allergy was found in 27 patients whereas GER was present in 53 patients.

Apnea was cleared in the entire group postoperatively. However, 9 patients complained of nasal obstruction, 16 patients had mouth breathing, 9 patients had snoring and 17 patients continued to have nasal discharge two months after the operation. Statistical assessment showed a significant difference for each symptom between the preand postoperative level $(\mathrm{p}<0.001)$.

In the re-examination of the patients with persistent symptoms, none of them were found to have adenoid vegetation greater than grade 1 . However, $71 \%$ of these patients had nasal pathology, 50\% had allergy and 50\% had GER.

Compared to symptom-free patients, patients with persistent obstructive symptoms (snoring, mouth breathing, nasal obstruction) were found to have significantly higher rates of allergy $(p<0.001)$ and nasal pathology $(p$ $<0.001)$, whereas, GER rate was similar $(\mathrm{p}=0.374)$.

In case of persistant nasal discharge, allergy $(\mathrm{p}=0.024)$ and GER ( $p=0.036)$ was found to be significantly more frequent.

Table 1. The incidence of pre- and postoperative symptoms of children with adenoid hypertrophy.

\begin{tabular}{ccc}
\hline \multirow{2}{*}{ Symptoms } & \multicolumn{2}{c}{ Present } \\
\cline { 2 - 3 } & $\begin{array}{r}\text { Preoperatively } \\
(\%=\mathrm{n})\end{array}$ & $\begin{array}{c}\text { Postoperatively } \\
(\%=\mathrm{n})\end{array}$ \\
\hline Apnea & 43 & 0 \\
Nasal obstruction & 85 & 9 \\
Mouth breathing & 95 & 16 \\
Snoring & 92 & 9 \\
Nasal discharge & 49 & 17 \\
\hline
\end{tabular}




\section{Discussions}

Adenoidectomy is an effective procedure for the relief of nasal obstruction, with a more than $75 \%$ satisfaction in our series. These findings are in line with the reported surgical success rates of $70 \%$ to $100 \%$ [3-7].

Twenty-two percent of the patients had persistent or recurrent symptoms. Most conspicuous cause of failure appeared to be nasal pathologies with the rate of $71 \%$. Obstructive symptoms, namely, mouth breathing, snoring and nasal obstruction may persist in these patients despite effective reduction of the adenoid tissue. Actually, recurrence of obstructive adenoid tissue reported to be the occasion in $19 \%$ of the treatment failures in the long-term [16]. However, in short-term, our findings stress the need for nasal endoscopy in the reevaluation of patients with persistent symptoms after adenoidectomy.

Hypertrophic tonsils may also be the causative factor of obstructive symptoms. In order to assess adenoidectomy only, we excluded the patients with grade 3-4 tonsillar hypertrophy. There are diverse opinions on the outcome of adenotonsillectomy or adenoidectomy alone for patients with upper airway obstruction. Shintani et al. [4] found no difference in success rate between adenoidectomy alone $(80 \%)$ and adenotonsillectomy $(84 \%)$ in children with upper airway obstruction. More recently, Shatz [17] described an excellent experience with adenoidectomy alone for infants with obstructive sleep apnea. Only one study found adenoidectomy alone to be an insufficient surgery [3]. The authors reported that $70 \%$ of their group of children with polysomnography-proven obstructive sleep apnea had already undergone adenoidectomy. Since the symptoms improved after tonsillectomy, they concluded that adenoidectomy alone "is not enough" However, their study was limited by a selection bias, in that the number of patients who improved with adenoidectomy alone was not mentioned. To be "on the safe side," many surgeons tend to perform adenoidectomy together with tonsillectomy for the treatment of obstructive upper airway disease $[2,18]$. In our center, however, as tonsillectomy has a higher morbidity and mortality rate than adenoidectomy, we opt for adenoidectomy alone in patients with obstructive sleep apnea and normal- or near-normal-sized tonsils (grade 1 - 2). Additional factors that influence the decision are patient age, symptom severity or polysomnographic findings, and the personal experience of the surgeon. A larger prospective randomized study comparing adenoidectomy to adenotonsillectomy is needed.

The prevalence of allergic rhinitis was reported as $15 \%-18 \%$ in Turkish children. $[19,20]$. In the present study, allergic rhinitis was confirmed in $27 \%$ of the patients with skin prick test or RAST, which is definitely greater than the expected rate. Moreover, allergy rate was significantly greater in patients with persistent obstructive symptoms and nasal drainage $[(\mathrm{p}=0,001),(\mathrm{p}=$ 0.024)].

GER was diagnosed in $51 \%$ of the patients in our study. GER was reported to be more prevalent in patients with adenoid hypertrophy (64.5\%) compared to normal children (25\%) [21]. Carr et al. [22] presented GER incidence as $42 \%$ in children under 2 years of age with adenoidectomy. These results support that pharyngeal acid reflux may play an important role in the etiology of adenoid hypertrophy.

However, GER was not established as a causative factor in the persistence or recurrence of obstructive symptoms in the short term $(\mathrm{p}=0.374)$. On the other hand, postoperative nasal drainage was significantly higher in patients with GER $(p=0.036)$.

\section{Conclusion}

Adenoidectomy alone is an effective treatment for nasal obstruction and obstructive sleep symptoms in children. Persistent symptoms are usually due to nasal pathology and allergy. Patients with persistent symptoms should be evaluated for these factors. In case of persistent nasal discharge, GER should also be considered in the differential diagnosis. Preoperative identificaiton of these factors might be contributory for improving outcomes of the adenoidectomy operation and also for proper patient counseling. Larger prospective studies are warranted to confirm long term effects of adenoidectomy and establish the failure resons.

\section{REFERENCES}

[1] C. D. Bluestone, "Current Indications for Tonsillectomy and Adenoidectomy," The Annals of Otology, Rhinology \& Laryngology, Vol. 101, No. 1, 1992, pp. 58-64.

[2] E. S. Deutsch, "Tonsillectomy and Adenoidectomy: Changing Indications," Pediatric Clinics of North America, Vol. 43, No. 6, 1996, pp. 1319-1338. doi:10.1016/S0031-3955(05)70521-6

[3] P. Nieminen, U. Tolonen and H. Lopponen, "Snoring and Obstructive Sleep Apnea in Children: A 6-Month Follow-Up Study," Archives of Otolaryngology, Head \& Neck Surgery, Vol. 126, No. 4, 2000, pp. 481-486.

[4] T. Shintani, K. Asakura and A. Kataura, "The Effect of Adenotonsillectomy in Children with OSA," International Journal of Pediatric Otorhinolaryngology, Vol. 44, No. 1, 1998, pp. 51-58. doi:10.1016/S0165-5876(98)00047-0

[5] M. Wolfensberger, J. A. Haury and T. Linder, "Parent Satisfaction 1 Year after Adenotonsillectomy of Their Children," International Journal of Pediatric Otorhinolaryngology, Vol. 56, No. 4, 2000, pp. 199-205. doi:10.1016/S0165-5876(00)00441-9

[6] L. M. De Serres, C. Derkay, K. Sie, et al., "Impact of 
Adenotonsillectomy on Quality of Life in Children with Obstructive Sleep Disorders," Archives of Otolaryngology, Head \& Neck Surgery, Vol. 128, No. 5, 2002, pp. 489-496.

[7] J. S. Suen, J. E. Arnold and L. J Brooks, "Adenotonsillectomy for Treatment of Obstructive Sleep Apnea in Children," Archives of Otolaryngology, Head \& Neck Surgery, Vol. 121, No. 5, 1995, pp. 525-530. doi:10.1001/archotol.1995.01890050023005

[8] R. M. Rosenfeld, "Pilot Study of Outcomes in Pediatric Rhinosinusitis," Archives of Otolaryngology, Head \& Neck Surgery, Vol. 121, No. 7, 1995, pp. 729-736. doi:10.1001/archotol.1995.01890070015005

[9] S. J. Vanderberg and D. G. Heatley, "Efficacy of Adenoidectomy in Relieving Symptoms of Chronic Sinusitis in Children," Archives of Otolaryngology, Head \& Neck Surgery, Vol. 123, No. 7, 1997, pp. 675-678. doi:10.1001/archotol.1997.01900070013002

[10] M. Modrzynski and E. Zawisza, "An Analysis of the Incidence of Adenoid Hypertrophy in Allergic Children," International Journal of Pediatric Otorhinolaryngology, Vol. 71, No. 5, 2007, pp. 713-719. doi:10.1016/j.ijporl.2006.12.018

[11] A. Tasker, P. W. Dettmar, M. Panetti, J. A. Koufman, J. Birchall and J. P. Pearson, "Is Gastric Reflux a Cause of Otitis Media with Effusion in Children?" The Laryngoscope, Vol. 112, No. 11, 2002, pp. 1930-1934. doi:10.1097/00005537-200211000-00004

[12] M. R. Bothwell, D. S. Parson, A. Talbot, G. J. Barbero and B. Wilder, "Outcome of Reflux Therapy on Pediatric Chronic Sinusitis," Otolaryngology-Head and Neck Surgery, Vol. 121, No. 3, 1999, pp. 255-262. doi:10.1016/S0194-5998(99)70181-6

[13] J. H. Cho, D. H. Lee and N. S. Lee, "Size Assessment of Adenoid and Nasopharyngeal Airway by Acoustic Rhinometry in Children," The Journal of Laryngology \& Otology, Vol. 113, No. 10, 1999, pp. 899-905 doi: $10.1017 / \mathrm{S} 0022215100145530$

[14] L. Brodsky, L. Moore and J. F. Stanievich, "A Comparison of Tonsillar Size and Oropharyngeal Dimensions in
Children with Obstructive Adenotonsillar Hypertrophy," International Journal of Pediatric Otorhinolaryngology, Vol. 13, No. 2, 1987, pp. 149-156. doi:10.1016/0165-5876(87)90091-7

[15] M. Friedman, H. Tanyeri, M. La Rosa, et al., "Clinical Predictors of Obstructive Sleep Apnea," The Laryngoscope, Vol. 109, No. 12, 1999, pp. 1901-1907. doi:10.1097/00005537-199912000-00002

[16] B. Joshua, G. Bahar, J. Sulkes, T. Shpitzer and E. Raveh, "Adenoidectomy: Long-Term Follow-Up," Otolaryngology—Head and Neck Surgery, Vol. 135, No. 4, 2006, pp. 576-580. doi:10.1016/j.otohns.2006.05.027

[17] A. Shatz, "Indications and Outcomes of Adenoidectomy in Infancy," The Annals of Otology, Rhinology, and Laryngology, Vol. 113, No. 10, 2004, pp. 835-838.

[18] D. H. Darrow and C. Siemens, "Indications for Tonsillectomy and Adenoidectomy," The Laryngoscope, Vol. 112, Suppl. 100, 2002, pp. 6-10. doi:10.1002/lary.5541121404

[19] C. Celtik, S. Okten, O. Okutan, H. Aydogdu, M. Bostancioglu, G. Ekuklu, A. Asan and M. Yazicioglu, "Investigation of Indoor Molds and Allergic Diseases in Public Primary Schools in Edirne City of Turkey," Asian Pacific Journal of Allergy and Immunology, Vol. 29, No. 1, 2011, pp. 42-49.

[20] E. Kurt, S. Metintas, I. Basyigit, I. Bulut, E. Coskun, S. Dabak, et al., "Prevalence and Risk Factors of Allergies in Turkey (PARFAIT): Results of a Multicentre CrossSectional Study in Adults," European Respiratory Journal, Vol. 33, No. 4, 2009, pp. 724-733. doi:10.1183/09031936.00082207

[21] B. Keles, K. Ozturk, H. Arbag, E. Gunel and B. Ozer, "Frequency of Pharyngeal Reflux in Children With Adenoid Hyperplasia," International Journal of Pediatric Otorhinolaryngology, Vol. 69, No. 6, 2005, pp. 1103-1107. doi:10.1016/j.ijporl.2005.02.019

[22] M. M. Carr, C. P. Poje, D. Ehrig and L. S. Brodsky, "Incidence of Reflux in Young Children Undergoing Adenoidectomy," The Laryngoscope, Vol. 111, No. 12, 2001, pp. 2170-2172. doi:10.1097/00005537-200112000-00018 